\title{
Der Osteopath - lebenslanges Lernen und Entwicklung
}

\author{
Kilian Dräger
}

Osteopathie ist Heilkunde, die den Menschen auf eine typisch systemische und integrative Weise betrachtet und behandelt und am ehesten ein allgemeinmedizinisches Indikationsspektrum abdeckt. Doch zum erfolgreichen Behandeln von Patienten braucht es auch kommunikative, psychologische, betriebswirtschaftliche und ethische Kompetenzen.

Dies verlangt eine gute Grundlage: profund ausgebildete Osteopathen, die sowohl kognitive als auch handwerkliche Fähigkeiten erworben haben. Osteopathen sollten systemisch und integrativ denken können, denn nur so werden die handwerklich erlernten Techniken adäquat an die individuelle Situation des Patienten angepasst. Doch auch wenn eine umfangreiche Ausbildung unverzichtbar ist, ist sie noch lange nicht hinreichend, um gut behandeln zu können.

\section{Progressive Einstellung}

Unverzichtbar ist insbesondere eine progressive Einstellung. Die meisten Osteopathen machen die Erfahrung, dass nach der Ausbildung der Lernprozess erst richtig interessant wird. Es ist geradezu so, dass man eine Ausbildung mit dem Verständnis beendet, nunmehr eingebettet in klinische Erfahrung, mit den guten und wesentlichen Fortbildungen beginnen zu können. Das ist durch-

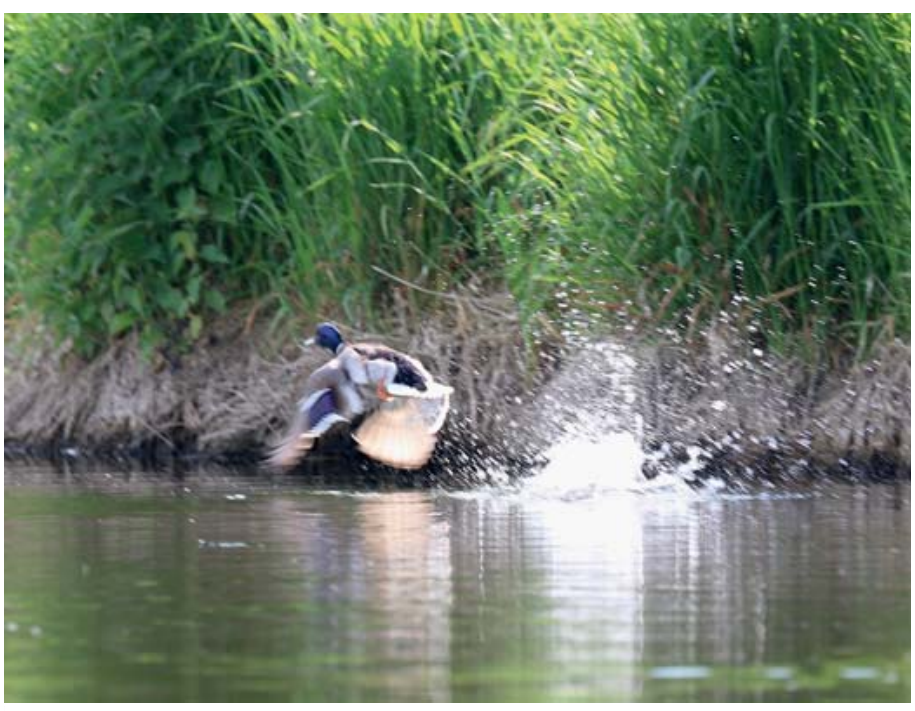

- Abb. 1 Staunen wir noch über uns vermeintlich bekannte Phänomene und fragen uns, wie es eigentlich sein kann, dass so etwas passiert? Wieso fliegt die Ente und kann aus dem Wasser starten? Oder wie können Kinder geboren werden? Wissen wir das wirklich? (৫ Kilian Dräger) aus mit dem Erlernen einer Fremdsprache zu vergleichen: Hat man die Vokabeln und Grammatik erfasst, fängt die Konversation erst richtig an. Gegen Ende der Osteopathieausbildung ahnt man, dass sich die vertraute Sichtweise und das bisherige therapeutische Vorgehen fortlaufend ändern werden. Es ist das stetig wachsende Verständnis für die Komplexität des Menschen, das die Notwendigkeit weiterer Forschung und Fortbildung erkennen lässt. Es ist der Geist der Neugier und des wiederkehrenden Aufbruchs, der die Trägheit und Selbstzufriedenheit überwinden muss ( $\bullet$ Abb. 1).

\section{Lernkultur}

In den Anfängen der Osteopathie in Deutschland gab es am Übergang von den 1980er- in die 1990er-Jahre eine Atmosphäre der Begeisterung für die Osteopathie, die mit reichlich Humor gespickt war. Wir brannten regelrecht und überschlugen uns im Interesse für diese besondere Methode, was das Lernen befeuerte und eigene Kreativität anregte. Es gab noch keine deutschsprachige Literatur - nur mündliche „Überlieferungen“ und wilde Sammlungen von Fotokopien. Der Anteil der praktischen Arbeit war sicher auch unstrukturierter und weniger differenziert als heute. Alles in allem gab es in den Schulen noch kein echtes didaktisches Konzept, sondern ein reichlich verworrenes Durcheinander. Auf den PostgraduateFortbildungen trafen sich viele interessante Persönlichkeiten, Originale, die keinen trockenen Stoff, sondern lebendige Osteopathie lehren oder lernen wollten. Abends feierten, musizierten und tanzten wir gemeinsam. 
Durch diese Lernkultur wurde unser Ausdruck vitaler und unsere Gewebe in andere Zustände und Dimensionen befördert und erweitert. Auch das sind Qualitäten, die im Leben und damit bei den Funktionen und Dysfunktionen der Gewebe eine bedeutende Rolle spielen. Pit Dijs sagte einmal: „Alle Gewebe haben eine Funktion und Dysfunktion - aber es sind auch alles Gewebe, die lachen und weinen können.“ [4]

\section{Selbstkritik}

Es ist glücklicherweise nicht so, dass diese besonderen Qualitäten bei osteopathischen Aus- und Fortbildungen heute nicht mehr zu finden wären, doch die Stimmung hat sich verändert. Einerseits hat sich die Vermittlung von Kompetenzen verbessert, andererseits birgt ein geebneter Weg auch die Gefahr, in einen behäbigen Trott zu verfallen. Die Pioniere von damals sind heute Lehrer, die ihre vielen, teils mühsam erworbenen Erkenntnisse und Erfahrungen in gut portionierten Häppchen weitergeben. Was in den 1990er-Jahren als verwirrend und schlecht durchschaubar erschien, wird heute überwiegend auf eine gute und klare Weise präsentiert.

Die verwirrte und verwirrende Vermittlung ist natürlich auch heute noch bei schlechten Ausbildungen anzutreffen. Es gibt Leute, die sich berufen fühlen, etwas weiter zu geben, obwohl sie selbst kaum eine Ahnung haben, worum es geht. Ich erinnere mich an einen „Lehrer“, der die „Wisbrett-Technik“ beschrieb. Gemeint war die „VSpread-Technik“, die man keinesfalls mit einem Brett, sondern dem Namen entsprechend V-spreizend ausführt. Einerseits ist dieses Missverständnis belustigend, andererseits ist der „Mut“, so offen seine Inkompetenz zur Schau zu stellen, beeindruckend. Ja, man kann davon aus- gehen, dass der „Lehrer“ seinen Fauxpas nicht bemerkt hat. Das sollte uns allen zu denken geben!

Wir können davon ausgehen, dass wir alle fehlerhafte Überzeugungen mit uns herumtragen, und sollten uns daher permanent selbstkritisch überprüfen und unsere Überzeugungen hinterfragen. Wir laufen sonst Gefahr, aus einer unsinnigen Kombination willkürlicher therapeutischer Ansätze abstruse Begriffe wie z. B. Trigger-OsteoPraktik zu kreieren. Hier werden Begriffe wie die Techniken von Triggerpunktbehandlung und Chiropraktik mit der Osteopathie gemischt, wobei die chirotherapeutischen Techniken und Triggerpunktbehandlung Bestandteil des osteopathischen Konzepts sind. Das zeugt allein von Unverständnis.

Große Lehrer können ein Vorbild dafür sein, wie man mit Fragen umgeht - offen für die Entdeckung von Neuem und Unbekanntem. Ann Wales, die Schülerin von W.G. Sutherland und Lehrerin von Rachel Brooks und Sue Turner, hat dies im hohen Alter von 98 Jahren schlicht und einfach demonstriert. Auf die Frage, wie denn die Technik der transversalen Fluktuation auszuführen sei, soll sie gesagt haben: „Oh, das habe ich vor kurzem verstanden.“ Man sollte die Kunst honorieren, so eine einfache Technik jahrzehntelang auszuführen und dann noch etwas Neues darin zu entdecken. Die Offenheit und Stärke sowie Unvollständigkeit und Unsicherheit des eigenen Therapievermögens auszuhalten, ermöglicht die Vertiefung des eigenen Verständnisses, wodurch die Technik präziser und effektiver angewandt werden kann.

So positiv die verbesserte didaktische Vermittlung zu bewerten ist, entsteht dadurch bei manchen Studenten die 


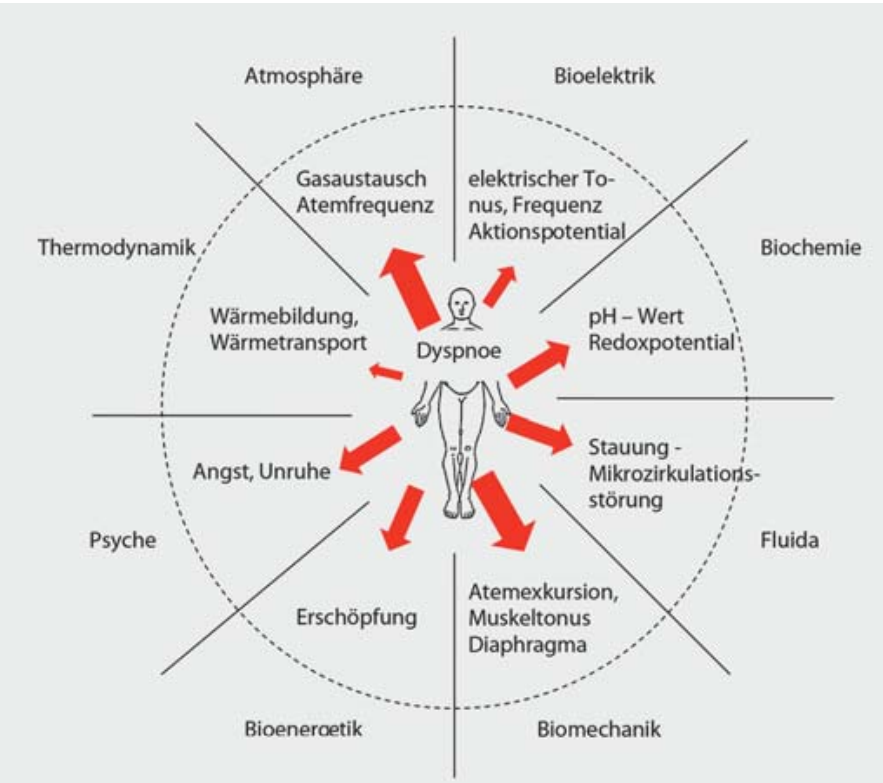

- Abb. 2 Verschiedene Dimensionen und individuelle Ausprägung des Individuums. (aus: [4])

Überzeugung, sie hätten schon alles durchdrungen. Gut mit Skripten bestückt, werden sie kritischer mit den Lehrern anstatt mit sich selbst und laufen Gefahr sich zu überschätzen. Dabei ist eine osteopathische Behandlung eher wie eine Annäherung, wie ein Dialog, indem man mit bestimmten Techniken in einem Gewebe vitale Reaktionen hervorrufen möchte. Dies gilt gleichermaßen für einen Trust, eine fasziale Technik und viszerale oder neurokraniale Techniken.

\section{Entwicklungsstadien}

Osteopathie ist keine Sammlung von Techniken. Still meinte sogar, dass er nie eine Technik vermittelt habe. Natürlich hat er Techniken als präzise Annäherung gelehrt, aber sie sind eben nur als Mittel zum Zweck des Dialogs zu verstehen und ein Dialog ist wie jedes Gespräch nie exakt zu wiederholen. Immer sollten die individuellen Besonderheiten wahrgenommen und berücksichtigt werden.

Es ist auch einleuchtend, dass die Fähigkeit, zunehmend mehr Besonderheiten zu erkennen und sich in den Techniken optimal an den Patienten anpassen zu können, von der aufgewendeten Zeit und Erfahrung abhängt wie auch von der offenen und fragenden, also forschenden Haltung. Ann Wales hat sich wohl in diesem Sinne als ständige Anfängerin begriffen, was von größter Fortschrittlichkeit zeugt.

Das führt mich direkt zu einem gehaltvollen Witz über die 4 Entwicklungsstadien eines Osteopathen: Das 1. Stadi- um eines Osteopathen ist die begründete Unsicherheit. Das 2. ist die unbegründete Sicherheit. Das 3. ist die begründete Sicherheit und das 4. die unbegründete Unsicherheit.

Während ein Anfänger begründet unsicher ist, stellt sich nach etwas Zeit die Überzeugung ein, etwas zu können, was zu deutlich mehr Selbstsicherheit und zunächst Selbstüberschätzung führt. Die Erkenntnis und Erfahrung sind noch nicht so tiefgreifend, dass die Sicherheit begründet wäre. Erst mit zunehmender Berufserfahrung wird die Sicherheit in Kenntnis und Ausübung so groß, dass sie auch aus Patientensicht als begründet angenommen werden kann. Doch ist das nicht das Ende der Entwicklung. Während die Sammlung von Erfahrungen und Forschungen weiter wächst, wächst auch die Erkenntnis, wie groß das zu bearbeitende Feld ist. Es ist schier nicht zu überschauen und letztlich nicht zu kontrollieren. Die Sicherheit der korrekt und kunstgerecht lege artis ausgeführten Techniken bleibt über die Zeit erhalten. Zusätzlich erwirbt man einen aufmerksamen Zweifel, weil die Antwort des Gewebes nicht zu kontrollieren ist und bei jeder Behandlung unerwartete Reaktionen aufzeigen kann. Auch wenn mit der Zeit die Erfahrung immer größer wird und aufgrund der großen "Gesprächserfahrung" die Verläufe oft gut einschätzbar sind, ist das klare Bewusstsein von den potenziellen, unkontrollierbaren und unvorhersehbaren Wendungen doch das weitest fortgeschrittene Stadium und somit Ziel der professionellen und persönlichen Entwicklung.

\section{Elemente der Heilung}

Es ist ein Segen, wenn sich diese Erkenntnis schon früh einstellt. Einige ältere Kollegen haben für mich eine vorbildliche Haltung eingenommen. Nach eigener Aussage haben sie diese Haltung erst gegen Ende ihrer Berufsausübung erworben. Es ist der leise Zweifel an den eigenen Fähigkeiten, der gepaart mit der professionellen Gewissheit die eigene Haltung verfeinert. Die Begründung dieser Haltung liegt aber im Patienten selbst: Es ist immer der Patient, der das wesentliche Element der Heilung ist. Das gilt für jeglichen Heilungsprozess. Trotz perfekt ausgeführter HVLA-Technik muss das Gewebe leben, um sich stabil integrieren und heilen zu können - Heilung findet selbst bei einer perfekt manipulierten Leiche nicht mehr statt. Die Aufgabe des Heilkundigen besteht darin, die Umstände so zu beeinflussen, dass der heilende Prozess stattfinden kann, und darin den Körper optimal zu unterstützen. Sicherheit in der bestmöglichen Therapieausübung und Ungewissheit in dem vom Körper beeinflussten Ergebnis scheinen mir die optimale Unterstützung für den Heilungsprozess eines Individuums zu sein. Osteopathen hinterfragen und kontrollieren bei der Behandlung, ob der Heilungsprozess günstig verläuft und korrigieren ihr Vorgehen bei Bedarf. 
Auch Ann Wales scheint bei ihren Behandlungen, die sie teilweise über Stunden ausgedehnt haben soll, das Quantum an vorbildlicher Unsicherheit mit sich geführt zu haben. Man führt eine Behandlung als Dialog so lange, bis der Patient für sich an den richtigen Punkt gekommen ist. Dies kann manchmal schnell gehen, aber manchmal braucht es auch lange, weil es nicht nur auf den „enorm qualifizierten“ Osteopathen ankommt, sondern im Wesentlichen auf den Patienten und sein Vermögen zu reagieren.

Da die menschliche Komplexität als offenes System unbegrenzt und unüberschaubar ist, fordert die osteopathische Methode im besonderen Maße zur ständigen Fortbildung heraus, um das Verständnis für die menschlichen Abläufe ständig zu erweitern.

\section{Systemische Interaktion}

Die verschiedenen Dimensionen des Menschen stehen untereinander in systemischer Wechselwirkung und machen ihn in seiner Gesamtheit aus ( $\bullet$ Abb. 2). Dies wird u. a. durch die Physiologie wissenschaftlich beschrieben, wobei die Osteopathie die funktionellen und psychophysikalischen Wechselwirkungen konsequent und direkt für den therapeutischen Einsatz ihrer speziellen Techniken nutzt. Die roten Pfeile in $\mathbf{A} \mathbf{A b} \mathbf{b} . \mathbf{2}$ stehen für eine unterschiedliche Dynamik der Dimensionen. Sie zeigen sowohl das individuelle Dysfunktionsmuster an als auch den Versuch, diese Dysbalancen dynamisch im Gleichgewicht zu halten. Je mehr Aspekte des Zusammenspiels wir berücksichtigen, umso mehr verstehen und begegnen wir dem Menschen als einem individuellen Ganzen. Solange der Mensch lebt, ist Balance zu finden.

Behandlungsziel ist die freie Bewegung in möglichst allen Dimensionen, wodurch sich eine freie Dynamik entfalten kann: im Allgemeinen, im Speziellen, im Gewebe, Organen und Funktionskreisläufen, sowie beim ganzen Menschen.

\section{Balance}

In der osteopathischen Ausbildung ist die Praxis und Selbsterfahrung ein elementarer Bestandteil, da Dialoge ein praktisches Können erfordern und nicht theoretisch erlernt werden können. Eine biomechanische Dysfunktion sollte biomechanisch angesprochen und gelöst werden können, bei einer emotionalen Dysfunktion braucht es die Fähigkeit, eben dort resonanzfähig und aktiv sein zu können. Elektromagnetische Vorgänge im Patienten stehen in Wechselwirkung mit elektromagnetischen Prozessen im Therapeuten (z. B. das elektromagnetische Feld des Herzens mit einem messbaren Radius von $2 \mathrm{~m}$ ).

Die Dysfunktionen der verschiedenen Dimensionen verschieben den Balancepunkt des Menschen und stellen überdies sein Funktionsmuster dar ( $\triangleright$ Abb. 2). Dies umfassend wahrnehmen und im Sinne einer Behandlung damit in „physikalische Resonanz" treten zu können, ist Grundlage der therapeutischen Tätigkeit. Während solche Dialoge beim Laien meist unbewusst erfolgen, muss man für therapeutische Ausbildungen fordern, dass hier klare Analysen den Untersuchungs- und Behandlungsmodus professionalisieren.

Dialoge sind nicht kontrollierbar oder vorhersehbar, genau wie Gespräche zwischen freien Partnern. Während des therapeutischen Prozesses sollte der Osteopath den Dialog aufnehmen können. Notwendig ist dazu Wahrnehmungsfähigkeit sowie weiterhin die Fähigkeit, eigene Impulse als Anregungen frei und produktiv einsetzen zu können. Dieser freie Einsatz von Behandlungsimpulsen ist von der eigenen Balance abhängig. Die eigene Balance ist ein Feld steter Übung und Arbeit an sich selbst.

Es scheint manchen Osteopathen unmöglich zu sein, eine balancierte, d. h. neutrale und objektive Haltung zu entwickeln. Zugegebenermaßen, dies mag schwierig bzw. höchst anspruchsvoll sein, aber es gibt keine akzeptable Alternative, da wir den goldenen Standard von randomisierten Doppelblindstudien nicht bei offenen physiologischen Prozessen in vivo einsetzen können. Studien sind auf bestimmte Fragestellungen hin geformt und zugespitzt, während komplexe menschliche Prozesse stets variablen Anforderungen durch die Umwelt ausgesetzt sind. Es ist unbedingt anzuerkennen, dass Leben in seiner Komplexität nun mal ein Prozess eines offenen Systems ist.

Um eine neutrale Haltung zu entwickeln, ist Balance nötig. Die Analyse der einfachen Grundgesetze, auf denen das Balance-Konzept beruht, zeigt, dass es sich lohnt, diese Grundgesetze für sich selbst zu erkunden.

Mittels Balance-Konzept [1, 2] kann man komplexe Systeme in einen einzigen Balancepunkt verdichten und der einfachen Interpretation zugänglich machen. Die Besonderheit des Konzepts ist die Synthese von logischem Verständnis und einfacher Wahrnehmung. Ratio und direkte wie subjektive Wahrnehmung stehen nicht mehr im Widerspruch zueinander. Es ist eine, wenn nicht sogar die Möglichkeit, komplexen Systemen geordnet zu begegnen und sie einfach zu handhaben. Die Erfahrung dieser Normalität, die einem natürlichen und freien Fluss der Prozesse entspricht, bedeutet Einfachheit. Eine Einfachheit, die im Gegensatz zur Trivialität eine meisterliche Fähigkeit ist.

\section{Kennen und Können}

Die Anerkennung dieses Umstands führt zu Demut, die auf klarer Sicherheit der Kompetenz ruht. So berichtet ein Schüler Rollin Beckers von einer ungewöhnlichen Unterrichtsbegebenheit. Dieser erhielt beim Stellen einer Frage als Antwort einen Stoß, der ihn gegen eine Glastür 
prallen ließ. Dabei ging es wohl um die Möglichkeit, durch den plötzlichen Impuls aus all seinen Gewohnheiten herauszufallen und in eine ungewohnte Normalität hineinzugleiten. Diese individuelle Unterrichtssituation ist vergleichbar mit einer Freisetzung von allen Hindernissen, wie man es einem Patienten wünschen würde. Glücklicherweise hat Beckers Schüler diesen Vorgang weder empört noch beleidigt, sondern überrascht aufgenommen. Er hat so viel Offenheit aufbringen können, dass er das Essenzielle des Impulses als Nachhall mit sich trägt. Er besteht in ihm weiter als ein wichtiges Ereignis in seinem Erlebnisraum und kann wegen dieser Wertschätzung wohl einfach im Verborgenen in ihm weiterarbeiten. Vielleicht arbeitet es weiter an seinem „Vorhängeschloss“, bis dieses endlich die Tür zum Aufspringen freigibt. Dabei kann man beim feinen und klaren Becker das eher seltene Vermögen meisterlichen Handelns vermuten.

Obwohl Rollin Becker einer der berühmtesten Lehrer ist, gab es anscheinend auch Schüler, die ihn nicht verstanden haben. Sie waren nicht bereit, ihr eigenes Denkmuster infrage zu stellen, ihre eigenen Vorannahmen aufzugeben und sich neuen Informationen, neuen Welten zu öffnen. Rollin Becker ist dafür bekannt, dass er besonders effektiv osteopathisch behandeln konnte und für Viele Türen geöffnet hat - zu einem größeren Verständnis und einem vergrößerten Behandlungspotenzial. Es ist erstrebenswert, sich fruchtbaren Hilfestellungen und Informationen zu öffnen, um diese in die eigene Professionalität und Menschlichkeit zu integrieren.

\section{Zugleich konkret und abstrakt}

Wenn auch diese weit fortgeschrittenen Fähigkeiten faszinierend sind, so bauen sie doch im professionellen Handeln auf einem Fundament des Kennens und Könnens auf. „Es ist noch kein Meister vom Himmel gefallen“, ist der Satz, mit dem man zum handwerklichen Üben aufgefordert wird. Es ist die Kunst eines Meisters, die das handwerkliche Können mit der höchsten Qualität ausstattet. Es ist der Griff eines Meisters, der ein Werkstück so fasst, dass alles richtig sitzt.

Was bedeutet das, wenn das Werkstück lebendiges Gewebe ist? Es ist die rätselhafte Balance, in die ein Mensch dann kommt, eine Balance die nichts Verfälschendes, nichts extra beinhaltet. Doch diese Einfachheit ist höchster Anspruch in einer Behandlung - zugleich konkret und abstrakt. Eine Frage bleibt steter Begleiter: Was ist neben einem therapeutischen Impuls wegzulassen, damit der therapeutische Prozess von einem Osteopathen fruchtbar begleitet oder auch geführt werden kann? Welche widersprüchlichen Impulse führe ich unwillkürlich und von mir selbst unbemerkt zeitgleich aus schlechter Angewohnheit aus? Diese widerstreitenden Impulse behindern den einfachen therapeutischen Prozess bzw. Dialog und sollten von uns weggelassen werden. Dies fordert einen Osteopathen, seine Dialogfähigkeit lebenslang zu erweitern.

Der Lernprozess in der Osteopathie ist Herausforderung und Chance für die ständige wissenschaftliche wie auch persönliche Entwicklung, oder wie man es psychologisch ausdrücken könnte, menschliche Reife. Es sind Werte wie Integrität der Persönlichkeit, Aufrichtigkeit und menschliche Wärme, die das Vertrauen des Patienten in den Therapeuten rechtfertigen.

In der Entwicklung und Pflege der Fähigkeiten von Wahrnehmung und Behandlung liegt eine große Verantwortung. Eine profunde Ausbildung und fortwährende Fortbildung sollten der Verantwortung gerecht werden und verpflichtend sein.

Autorinnen/Autoren

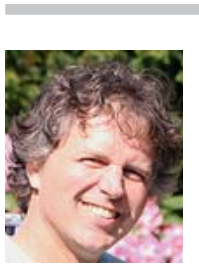
Kilian Dräger

ist Arzt, Osteopath, Physio- und Shiatsu-Therapeut. Er ist seit 1995 auch als Dozent tätig, zudem ist er Fakultätsmitglied des SCCO und Lehrbeauftragter der medizinischen Fakultät der Uni Hamburg. Er setzt sich für die Osteopathie als Vorstand der osteopathischen Kindersprechstunde, der DÄGO und des BDOÄ ein. Er hat mehrere osteopathische Fachartikel und ein Buch veröffentlicht.

Korrespondenzadresse

Dr. med. Kilian Dräger D.o.

Beim Andreasbrunnen 7

20249 Hamburg

draeger@daego.de

Literatur

[1] Dräger K. Balance-Konzept - Grundlagen und Fallbeispiele. OM 2015; 4: 17-22

[2] Dräger K. Grundgesetz und Vollendung der Bewegung. DO 2017; 3: 24-30

[3] Dräger K, Dräger C. Beziehung und Bindung in der osteopathischen Kinderbehandlung. OM 2009; 4: 18-22

[4] Dräger K, Van Den Heede P, Kleeßen H. Osteopathie - Architektur der Balance. München: Elsevier; 2011

Bibliografie

DOI https://doi.org/10.1055/a-0651-8566

DO - Deutsche Zeitschrift für Osteopathie 2018; 16: 4-8

(c) Georg Thieme Verlag KG Stuttgart · New York ISSN 1610-5044 